Check for updates

Cite this: Soft Matter, 2018, 14,4615

Received 15th February 2018 Accepted 21st April 2018

DOI: $10.1039 / \mathrm{c} 8 \mathrm{sm} 00327 \mathrm{k}$

rsc.li/soft-matter-journal

\section{Phase behaviour and applications of a binary liquid mixture of methanol and a thermotropic liquid crystal $\dagger$}

\author{
Luis A. Serrano, (D) $\ddagger^{a}$ Maximiliano J. Fornerod, (D) $\ddagger^{a}$ Ye Yang, (D) ${ }^{a}$ \\ Simon Gaisford, (D) ${ }^{b}$ Francesco Stellacci (D) ${ }^{c}$ and Stefan Guldin (D) *a
}

\begin{abstract}
Herein, we report on the phase behaviour of a binary liquid mixture composed of methanol $(\mathrm{MeOH})$ and the thermotropic liquid crystal 4-cyano-4'-pentylbiphenyl (5CB). The corresponding phase diagram combines features of a conventional liquid-liquid mixture with characteristics that are particular to the nematic liquid crystal. We observe four arrangements as a function of composition and temperature, namely monophasic isotropic, monophasic nematic, biphasic isotropic-isotropic and biphasic isotropic-nematic, with an upper critical solution temperature of $24.4 \pm 0.5{ }^{\circ} \mathrm{C}$. The interplay of nematogenic and non-nematogenic species offers tunability of phase mixing and phase composition in an accessible temperature window and provides novel routes for the extraction of target compounds, here exemplarily shown for Crystal Violet, Doxorubicin, Eosin Y, Rhodamine 6G and Sudan IV.
\end{abstract}

\section{Introduction}

The phase behaviour of a binary liquid mixture is generally described by the Gibbs free energy of mixing. A phase diagram for partially miscible mixtures may be presented as a function of composition $\phi$ and temperature $T$, where the coexistence curve separates the monophasic and biphasic regions. ${ }^{1}$ The upper critical solution temperature (UCST) distinguishes the temperature above which the two liquids are completely miscible for all compositions from where phase separation occurs for certain compositions. The lower critical solution temperature (LCST) relates to an opposite scenario, where a single phase exists for all compositions below a certain temperature. An example of a binary mixture with an UCST is nitrobenzene and hexane, whereas water and triethylamine exhibit an LCST. ${ }^{2}$ We refer to a monograph by Francis for a comprehensive overview of binary liquid mixtures and their critical solution temperatures. ${ }^{3}$

Such binary liquid mixtures are of great importance for a variety of applications, including catalyst separation, ${ }^{4}$ chemical analysis $^{5}$ and microfluidics. ${ }^{6}$ Aqueous-organic systems find

\footnotetext{
${ }^{a}$ University College London, Department of Chemical Engineering, Torrington Place, London, WC1E 7JE, UK. E-mail: s.guldin@ucl.ac.uk

${ }^{b}$ University College London, School of Pharmacy, 29-39 Brunswick Square, London, WC1N 1AX, UK

'École Polytechnique Fédérale de Lausanne, Institute of Materials, Lausanne 1015, Switzerland

$\dagger$ Electronic supplementary information (ESI) available. See DOI: 10.1039/ c8sm00327k

\$ These authors contributed equally to this work.
}

industrial applications ranging from water pollution abatement to removal of waste petroleum and pesticides from soil. ${ }^{7}$ In contrast, aqueous two-phase systems (ATPSs) are formed of two aqueous phases and offer advantages related to their environmental sustainability and compatibility with biological media. ATPSs are typically composed of an upper phase that contains a high concentration of a more hydrophobic polymer such as polyethylene glycol and a lower phase with a denser and more hydrophilic polymer such as dextran. ${ }^{8,9}$ Other phase configurations include polymer-salt, salt-salt and combinations with ionic liquids. ${ }^{10,11}$ Binary liquid mixtures with an LCST are a promising material system for artificial microswimmers. In a recent study, a medium composed of a mixture of water and lutidine enabled triggering of active Brownian motion by spatially confined phase separation through local heating by a light stimulus. ${ }^{12}$ These findings prompted a number of interesting concepts towards active soft matter. ${ }^{13}$

In this work, we study the behaviour of a thermotropic liquid crystal (LC) in binary liquid mixtures. LCs are an important class of soft materials with a broad range of applications in optoelectronic and biomedical devices. ${ }^{14-17}$ Thermotropic LCs display temperature-dependent liquid crystalline mesophases with characteristic orientational and positional order of mesogens. In a nematic arrangement, the mesogens exhibit long-range orientational without positional order. ${ }^{18} \mathrm{~A}$ widely used nematic LC is 4-cyano-4'-pentylbiphenyl (5CB), which was first synthesised by Gray et al. in $1973 .{ }^{19}$ CCB undergoes a transition from crystalline to nematic at $24{ }^{\circ} \mathrm{C}$ and from nematic to isotropic at $35{ }^{\circ} \mathrm{C} .{ }^{19}$ 
The mixing of nematic LCs with non-nematogenic species, in particular polymers and nanoparticles, has been studied in both theory and experiments. ${ }^{20-26}$ Early work focused on elucidating the effect of non-nematogenic doping on the isotropic-to-nematic phase transition temperature $T_{\mathrm{I}-\mathrm{N} \cdot}{ }^{27}$ The phase behaviour of binary mixtures of low molecular weight nematic LCs and nonnematogenic species is typically well described by hybrid mean field models that take into account aspects of the Maier-Saupe as well as the Flory-Huggins theory. ${ }^{28-32}$ For a binary liquid mixture of a nematic LC and an isotropic liquid, the consideration of nematohydrodynamics enables to predict the kinetic effects of the phase separation. ${ }^{33}$

Herein, we report on the phase behaviour of a binary liquid mixture composed of $\mathrm{MeOH}$ and the nematic LC 5CB. This binary mixture is an important model system that deserves a detailed study. First, 5CB is a widely used nematic LC that exhibits its mesophase transitions near ambient conditions. Second, $\mathrm{MeOH}$ is a widely used organic solvent with a minimal molecular weight and thus ideally suited for studying dilution effects with non-nematogenic species. Furthermore, the interplay of $\mathrm{MeOH}$ as a versatile organic solvent and $5 \mathrm{CB}$ as a thermotropic LC solvent promises avenues of temperature-driven liquid-liquid extraction.

\section{Experimental}

Reagents: 5CB was obtained from Synthon Chemicals (99.5\% (GC)). Eosin Y (99\%), Doxorubicin hydrochloride (98-102\%, HPLC), Rhodamine 6G (99\%), Crystal Violet (for microscopy (Bact., Bot., Hist., Vit.)), Sudan IV (>80\%) and MeOH (HPLC grade) were purchased from Sigma Aldrich. All compounds were used without further purification.

Phase diagram: the $T-\phi$ phase diagram was experimentally mapped as follows. The corresponding volumes of 5CB and $\mathrm{MeOH}$ were placed in a $3.5 \mathrm{ml}$ quartz cuvette with a small magnetic stir bar and sealed by a cap. The mixtures were then heated to $40{ }^{\circ} \mathrm{C}$ and stirred at $1000 \mathrm{rpm}$ for $10 \mathrm{~min}$ to ensure that the solution was clear and homogeneous. Then, the solution was cooled down from $40{ }^{\circ} \mathrm{C}$ to $2{ }^{\circ} \mathrm{C}$ in steps of $5{ }^{\circ} \mathrm{C}$ and a dwell time of $3 \mathrm{~min}$ at each step. Once a first estimate was established by the cloud point method, the experiment was repeated in intervals of $1{ }^{\circ} \mathrm{C}$. The temperature and stirring were controlled by a Peltierregulated sample compartment (Quantum Northwest, QPod 2e). Videos were recorded using a digital microscope (Dino-Lite) that was attached to the optical port of the compartment; see Fig. S1 in the $\mathrm{ESI} \dagger$ for a schematic of the experimental set-up.

Absorbance measurements: the optical set-up consisted of a broadband laser-driven light source (Energetiq, EQ-99XFC), a temperature-controlled cuvette holder (Quantum Northwest, QPod 2e) and a high-sensitivity spectrometer (Ocean Optics, QE Pro). Optical fibres with a core diameter of $1000 \mu \mathrm{m}$ (Ocean Optics) were used for light transmission. Disposable polystyrene $4.5 \mathrm{ml}$ cuvettes (Fisher Scientific) served as sample compartments. The concentration of the molecules was $10 \mu \mathrm{M}$ except for Crystal Violet $(5 \mu \mathrm{M})$ and for Sudan IV $(20 \mu \mathrm{M})$.

\section{Extraction experiments}

The standard protocol involved dissolving a compound in $\mathrm{MeOH}$, adding an equal volume of $5 \mathrm{CB}$, heating at $40{ }^{\circ} \mathrm{C}$ for 5 min, manually shaking the vial to mix both phases and then leaving the mixture at $40{ }^{\circ} \mathrm{C}$ for $1 \mathrm{~min}$. Afterwards, the vial was cooled down to the desired temperature. Each of the phases was subsequently extracted and their absorbance was measured using the optical set-up described above.

Compositional analysis of $\mathrm{MeOH}-5 \mathrm{CB}$ mixtures: solutions of LCs in $\mathrm{MeOH}$ were prepared with the concentration of $5 \mathrm{CB}$ ranging from $5 \times 10^{-7} \mathrm{M}$ to $5 \times 10^{-5} \mathrm{M}$. The absorbance of the respective solutions was measured at $297 \mathrm{~nm}$, where the absorbance of $\mathrm{MeOH}$ was found to be negligible. The corresponding absorbance-based calibration curve for the $5 \mathrm{CB}$ concentration is shown in the $\mathrm{ESI} \dagger$ (Fig. S2). For validation of the phase composition, volume fractions were extracted from the respective phases and diluted with $\mathrm{MeOH}\left(10^{6} \times\right)$ to ensure that the concentration of $5 \mathrm{CB}$ would fall in the range of the calibration curve. Then, the absorbance of the diluted samples was measured and used to calculate the actual amount of 5CB in the sample.

Differential scanning calorimetry (DSC): characterisation was carried out on a Multi-Cell DSC (TA Instruments). Binary liquid mixtures of $0.4 \mathrm{ml}$ total volume and $0-50 \mathrm{vol} \% \mathrm{MeOH}$ were characterised in $1.0 \mathrm{ml}$ capacity ampoules with an empty cell as a reference. The samples were first heated to $50{ }^{\circ} \mathrm{C}$ before a cooling scan was conducted to $-30{ }^{\circ} \mathrm{C}$ at a ramp rate of $0.1{ }^{\circ} \mathrm{C} \mathrm{min}^{-1}$.

\section{Results}

The experimental set-up for the macroscopic examination of the phase behaviour is shown in the ESI $\dagger$ (Fig. S1). Samples were studied in a quartz cuvette inside a Peltier-controlled sample compartment and imaged in a side-view using a digital microscope. The experimental determination of the phase coexistence equilibrium as a function of composition was based on the cloud point method. Cooling of the monophasic solution was carried out until a transient cloudiness was observed, which was related to microscopic heterogeneity, evidenced by light being scattered by the refractive index contrast of the microphases. ${ }^{34}$ A typical temperature-dependent phase behaviour of a binary mixture of 50 vol\% $5 \mathrm{CB}$ and $50 \mathrm{vol} \% \mathrm{MeOH}$ is shown in Fig. 1 . At $40{ }^{\circ} \mathrm{C}$, the mixture was fully miscible and the system showed a single clear phase that remained monophasic over time (Fig. 1A). Upon cooling, the system eventually reached a cloud point at $25{ }^{\circ} \mathrm{C}$. When the solution was settled below the cloud point, the system subsequently separated into two clear phases (Fig. 1B). Finally, once cooled below $-1{ }^{\circ} \mathrm{C}$, the bottom phase became cloudy again (Fig. 1C). While the phase separation induced a temporary cloudiness related to a transient emulsification of the mixture, the cloudiness below $-1{ }^{\circ} \mathrm{C}$ remained, indicating a change of mesogen orientation to the nematic state. ${ }^{18}$

Further evidence is provided in the ESI. $\dagger$ In Fig. S3, ESI $\dagger$ the isotropic-to-nematic phase transition of undiluted 5CB is shown. In Fig. S4, ESI $\dagger$ a kinetic study is presented that documents the transient cloudiness imposed by the phase separation of the 

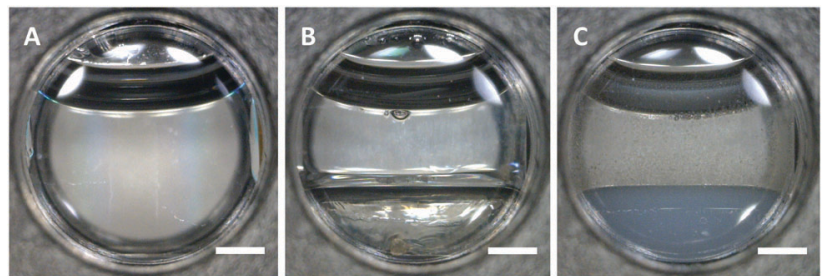

Fig. 1 Phase behaviour of a 50:50 vol\% mixture of $\mathrm{MeOH}$ and 5CB (A) $40{ }^{\circ} \mathrm{C}$ : above the UCST a single isotropic phase is observed. (B) $12{ }^{\circ} \mathrm{C}$ : below the UCST the mixture is phase separated with two clear phases. (C) $-2{ }^{\circ} \mathrm{C}$ : further cooling results in an opaque nematic bottom phase Scale bars represent $2 \mathrm{~mm}$. Note that the samples are shown in a side-view with a white backscreen for clarity.

5CB-MeOH mixture. In Fig. S5, ESI $\dagger$ a mixture of 50 vol\% $\mathrm{MeOH}$ and 50 vol\% $5 \mathrm{CB}$ is studied by optical microscopy in an LC cell under bright field and cross-polarised illumination. The microscopic observations are in line with the macroscopic behaviour, i.e. the appearance of two isotropic phases below $25{ }^{\circ} \mathrm{C}$ and the subsequent change to a biphasic isotropic-nematic system at $-1{ }^{\circ} \mathrm{C}$. It is worth noting that when reheating the biphasic 5CB-MeOH mixture to just above the cloud point (i.e. from $25^{\circ} \mathrm{C}$ to $26{ }^{\circ} \mathrm{C}$ ) under stirring, the mixture became a single phase and cleared within seconds.

Different mixtures of $5 \mathrm{CB}$ and $\mathrm{MeOH}$ were studied, and the results are summarised in a $T-\phi$ phase diagram shown in Fig. 2. The corresponding phase diagram in molar fractions is shown in the ESI $\dagger$ (Fig. S6). Alongside the diagram, all observed configurations are illustrated in a schematic. The phase diagram depicts four well-defined regions. The red diamonds represent the experimental results for phase separation. A corresponding phase coexistence curve with an upper critical solution temperature of $24.4 \pm 0.5{ }^{\circ} \mathrm{C}$ was derived by a second-degree polynomial interpolation of the experimental results. The black circles separate conditions where a nematic phase was observed from conditions that generated only isotropic configurations. Above the phase coexistence curve, a single isotropic phase (I) was found for all compositions with $\mathrm{MeOH}>13 \mathrm{vol} \%$. For a lower $\mathrm{MeOH}$ content and temperatures above the phase coexistence curve, a single nematic phase $(\mathrm{N})$ was observed. Below the phase coexistence curve, two biphasic regions were found, an isotropicisotropic $(\mathrm{I}+\mathrm{I})$ arrangement and a nematic-isotropic $(\mathrm{N}+\mathrm{I})$ arrangement. In the I + I region, two isotropic phases coexisted, namely $\mathrm{MeOH}$ enriched with 5CB and isotropic 5CB enriched with $\mathrm{MeOH}$, while in the $\mathrm{N}+\mathrm{I}$ region the 5CB-rich phase was nematic. In the course of this study, we did not experimentally observe a biphasic region at the boundary between the $\mathrm{N}$ and I phases and nor the co-existence of three phases which was expected at around $-1{ }^{\circ} \mathrm{C}$ and $13 \mathrm{vol} \% \mathrm{MeOH}$.

The phase diagram was further validated by comparing the expected phase composition from applying the lever rule to the actual composition of the phase separated sample for a given temperature. As shown in the $\mathrm{ESI}_{\dagger} \dagger$ (Fig. S2), 5CB exhibits a characteristic absorbance at $297 \mathrm{~nm}$, which allows the quantification of the composition of each phase via multiple dilution steps with $\mathrm{MeOH}$. In all cases the determined phase composition
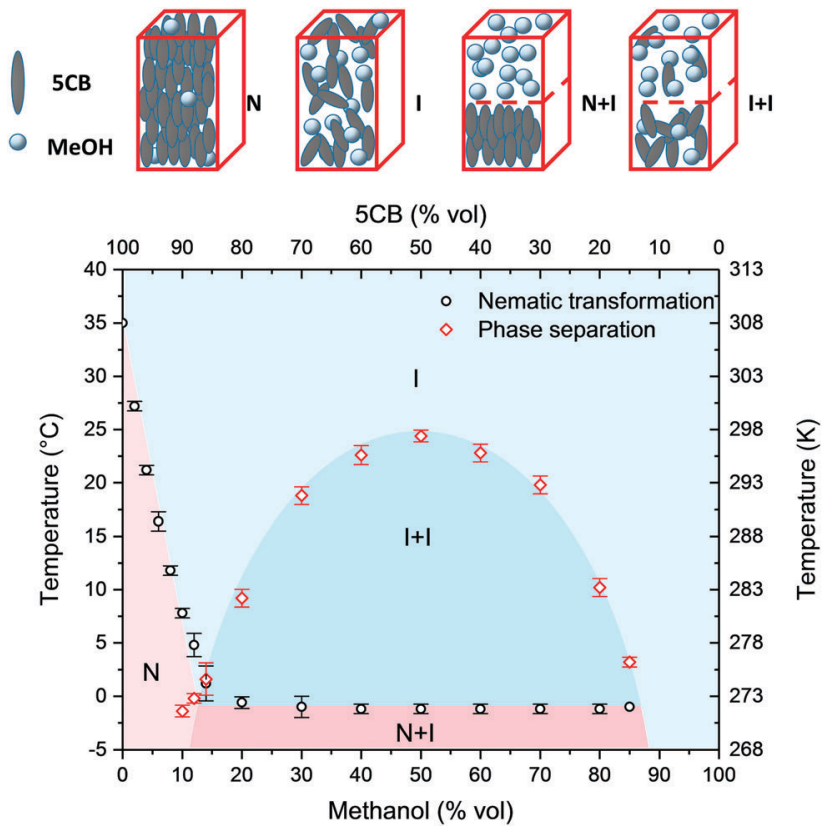

Fig. $2 \mathrm{~T}-\phi$ phase diagram of $5 \mathrm{CB}-\mathrm{MeOH}$ binary mixtures. The phase space is divided into four zones: isotropic (I), nematic (N), isotropic + isotropic $(\mathrm{I}+\mathrm{I})$ and nematic + isotropic $(\mathrm{N}+\mathrm{I})$. A schematic overview of the four arrangements is shown above.

was within $5 \%$ of the predicted composition based on the phase diagram (Table S2, ESI $\dagger$ ).

The phase diagram exhibits a number of interesting aspects. As indicated by the black circles, the isotropic-to-nematic phase transition $\left(T_{\mathrm{I}-\mathrm{N}}\right)$ is highly dependent on the $\mathrm{MeOH}$ content for compositions below $13 \% \mathrm{MeOH}$. We were able to tune the transition temperature systematically from $35{ }^{\circ} \mathrm{C}$ to $1{ }^{\circ} \mathrm{C}$ by an increasing addition of $\mathrm{MeOH}$. These results are in line with differential scanning calorimetry experiments shown in the ESI $\dagger$ (Fig. S7). When plotted in molar fractions (Fig. S6, ESI $\dagger$ ), a linear relationship for the tunability of $T_{\mathrm{I}-\mathrm{N}}$ by dilution with $\mathrm{MeOH}$ becomes evident. The observed behaviour is attributed to the so-called dilution effect of the nematic mean field ordering. ${ }^{24,35}$ Early work on the effect of solvents on the properties of nematic LCs reported a linear decrease at low solvent content. ${ }^{27}$ Doping with non-nematogenic solvent species was later proposed as a method to bi-directionally tune $T_{\mathrm{I}-\mathrm{N}}$ within a limited temperature range: lowering $T_{\mathrm{I}-\mathrm{N}}$ by dilution as well as increasing $T_{\mathrm{I}-\mathrm{N}}$ through donor-acceptor interactions of nematic mesogens and solvent molecules. ${ }^{36}$ More recently, a number of studies have investigated the effect of non-mesogenic impurities on the phase behaviour of 4-cyano-4'-octylbiphenyl (8CB). ${ }^{37,38}$ In the herein presented study, the linear decay of $T_{\mathrm{I}-\mathrm{N}}$ remained valid up to a molar fraction for the non-nematogenic impurity of 0.5 , enabling us to tune $T_{\mathrm{I}-\mathrm{N}}$ over a wide temperature range.

For a $\mathrm{MeOH}$ composition of $13 \mathrm{vol} \%$ and above, no further decrease was found. The observed horizontal phase boundary between $\mathrm{N}+\mathrm{I}$ and $\mathrm{I}+\mathrm{I}$ at a higher $\mathrm{MeOH}$ content indicates an upper limit of non-nematogenic volume content that can be accommodated by the nematic phase. Any additional increment in the $\mathrm{MeOH}$ content resulted in an expulsion from the nematic 
phase and thus the formation of a separate $\mathrm{MeOH}$-rich isotropic phase. We did not observe recrystallisation for any of the compositions in the studied temperature range $\left(-5{ }^{\circ} \mathrm{C}\right.$ to $\left.40{ }^{\circ} \mathrm{C}\right)$.

In a separate experiment, the dilution of $5 \mathrm{CB}$ with $\mathrm{CCl}_{4}$ was studied. As shown in the ESI $\dagger$ (Fig. S8), a similar trend was obtained with a further pronounced linear perturbation of $T_{\mathrm{I}-\mathrm{N}}$, indicating that the observed dilution effects are related to the spherical nature of $\mathrm{MeOH}$ to disturb the effective anisotropy rather than hydrogen-bonding between $\mathrm{MeOH}$ and 5CB.

The general shape and arrangement of the phase diagram are of further interest. When plotted in volume fractions, the single/two-phase coexistence curve resembles a conventional symmetric shape of a binary liquid mixture with partial miscibility. We found the phase space to be superimposed by the nematogenic behaviour of $5 \mathrm{CB}$, evidenced by an I-N phase boundary below $13 \mathrm{vol} \% \mathrm{MeOH}$ and a biphasic $\mathrm{N}+\mathrm{I}$ region at a higher $\mathrm{MeOH}$ content below $-1{ }^{\circ} \mathrm{C}$. These experimental results are well-aligned with earlier theoretical work on binary mixtures of nematic LCs with non-nematogenic solvents based on a combined model of Flory-Huggins and Maier-Saupe. ${ }^{32}$ The fact that we see a near linear decrease in the isotropic-to-nematic phase transition of 5CB at a lower and fully symmetric phase coexistence curve at a higher $\mathrm{MeOH}$ content suggests that the extremely low molecular weight $\mathrm{MeOH}$ acts as an ideal dilutant of $5 \mathrm{CB}$. At low volume content, it homogeneously blends and suppresses the isotropicto-nematic transition. Above $13 \mathrm{vol} \% \mathrm{MeOH}$, both components act as a near ideal mixture with full symmetry around an equal volume composition.

In order to understand the detailed interplay of 5CB and $\mathrm{MeOH}$ for the observed phase behaviour, further binary mixtures of nematogenic and non-nematogenic compounds with $\mathrm{MeOH}$ were studied, as shown in the ESI $\dagger$ (Fig. S9). An increase in the alkyl chain length in the case of 4-cyano-4'-hexylbiphenyl (6CB) led to a somewhat similar phase behaviour with a temperature offset of $6{ }^{\circ} \mathrm{C}$. In contrast to $5 \mathrm{CB}$, solidification was observed in the studied temperature range. The use of a non-nematogenic analogue to $5 \mathrm{CB}$ with a branched alkyl chain, namely $(S)$-4-cyano4'-(2-methylbutyl)biphenyl (CB15), led to a symmetric phase diagram with I and I + I phase arrangements and an UCST of $3{ }^{\circ} \mathrm{C}$.

Based on our findings of temperature-dictated phase behaviour and compositional tuning, the potential of using a $5 \mathrm{CB}-\mathrm{MeOH}$ binary mixture for temperature-driven liquid-liquid extraction was further explored. A series of molecules with different degrees of planarity, ranging from food colourants to chemotherapeutic drugs, were chosen for a comparative study as shown in Fig. 3.

A typical phase transfer experiment was carried out as follows: the compounds were individually dissolved in $\mathrm{MeOH}$ $(1 \mathrm{ml})$ and the solution was then transferred to a vial containing an equal volume of $5 \mathrm{CB}$. The samples were then placed in an oven at $40{ }^{\circ} \mathrm{C}$ for $5 \mathrm{~min}$, manually shaken for 5 seconds and further heated in the oven for $1 \mathrm{~min}$. Subsequently, the vials were placed in a fridge $\left(T=3.5{ }^{\circ} \mathrm{C}\right)$ for $10 \mathrm{~min}$ in order to allow the phases to separate and reach equilibrium. Finally, both the upper phase (MeOH-rich) and the bottom phase (5CB-rich) were extracted, and the absorbance maximum of each of the

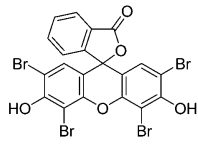

Eosin $Y(E O)$

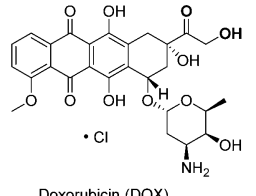

Doxorubicin (DOX)

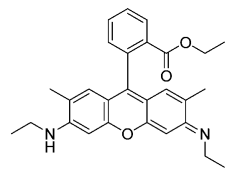

Rhodamine $6 \mathrm{G}$ (R6G)
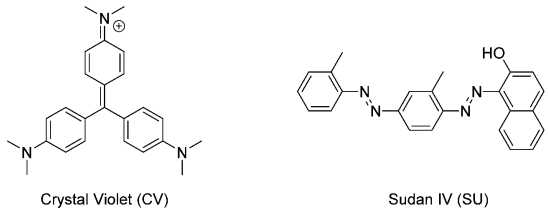

Sudan IV (SU)

Fig. 3 Molecular structure of model compounds for phase extraction. From left to right: Eosin Y, Crystal Violet, Doxorubicin, Sudan IV, and Rhodamine 6G.

phases was measured and compared to the original absorbance maximum of the compounds in $\mathrm{MeOH}$ in order to calculate the partition coefficients. Note that the maximum in absorbance was shifted to longer wavelengths $(5-10 \mathrm{~nm})$ for the 5CB-rich phase. We refer to the ESI $\dagger$ (Fig. S10) for the photographs of the vials at each step of the phase transfer experiment.

The results of the partitioning experiments are summarised in Fig. 4. Eosin $\mathrm{Y}$ and Doxorubicin showed partitioning predominantly into the $\mathrm{MeOH}$ phase with ratios of $4.9: 1$ and $3.2: 1$, respectively. Rhodamine $6 \mathrm{G}$ was found in equal amounts in both phases $(1.0: 1)$. In contrast, Crystal Violet and Sudan IV showed a strong tendency towards $5 \mathrm{CB}$, with partitioning ratios of $2.6: 1$ and $30: 1$, respectively. These results demonstrate that the chemical structure of the molecules had a dramatic effect on the partitioning between the 5CB-rich and $\mathrm{MeOH}$-rich phases. When rationalising the behaviour based on the molecular structures shown in Fig. 3, two properties come to mind, namely the polarity and the ability for $\pi-\pi$ stacking. Polarity is not likely to be the main driving force as only two compounds contained charges among the five chosen ones, namely Eosin $\mathrm{Y}$ and Doxorubicin, and these displayed opposite partitioning

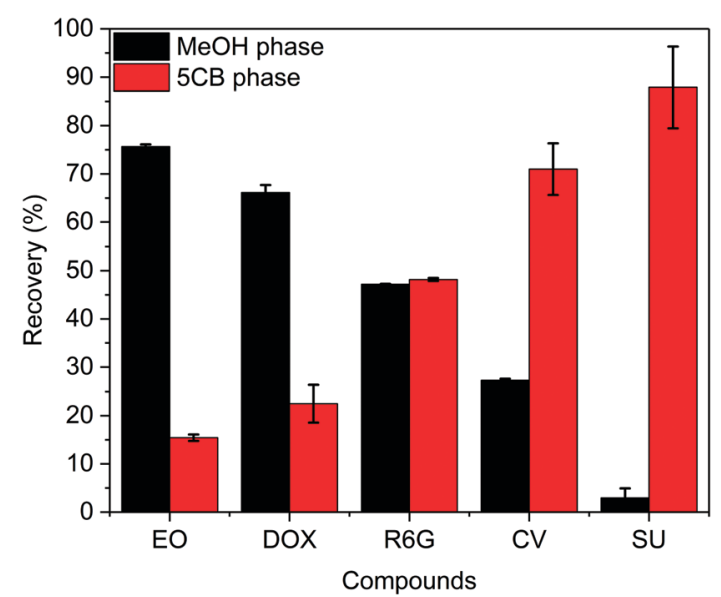

Fig. 4 Partitioning of model compounds. Percentage of Eosin $Y$ (EO), Doxorubicin (DOX), Rhodamine 6G $(\mathrm{RH})$, Crystal Violet $(\mathrm{CV})$ and Sudan IV (SU) extracted into the $5 \mathrm{CB}$ and $\mathrm{MeOH}$ phases at $3.5^{\circ} \mathrm{C}$, respectively. 
characteristics. On the other hand, the ability for $\pi-\pi$ stacking is associated with aromaticity and may be rationalised by Huckel's rule. ${ }^{39,40}$ The criteria for Huckel's rule were met by the five molecules in this study, as they all displayed at least one benzene moiety. The molecule that showed the highest affinity for the 5CB phase was Sudan IV. It is not only a planar molecule, ${ }^{41}$ but also the only one that contains an aromatic moiety (naphthalene) bigger than benzene. Thus, this molecule is most likely to form $\pi-\pi$ stacking. While Crystal Violet is not completely planar due to the steric hindrance of its aromatic rings, it still displayed the second highest affinity for the 5CBrich phase. It is worth noting that both Sudan IV and Crystal Violet contain electron-rich aromatic rings due to their azo and amino substituents, respectively. This is likely to enhance their affinity for $5 \mathrm{CB}$, since the aromatic core of $5 \mathrm{CB}$ is expected to be slightly electron deficient due to the electron-withdrawing character of the carbonitrile group. Interestingly, Rhodamine 6G was distributed in equal amounts in both phases. This molecule exhibits an orthogonal substituent which disrupts the ability of the molecule for $\pi-\pi$ stacking. ${ }^{42}$ This distortion is enhanced since the orthogonal aromatic ring contains an orthosubstituent. Following this trend, Doxorubicin is substituted with a bulky sugar ring, ${ }^{43}$ disrupting even more its ability for $\pi-\pi$ stacking. Finally, Eosin Y was found to have the highest affinity for the $\mathrm{MeOH}$ phase. This molecule is completely orthogonal and therefore very unlikely to $\pi$-stack.

According to the phase diagram of the binary mixture, the composition of both phases upon cooling and phase separation is highly temperature-dependent. Studying the effect of temperature on the partitioning of target molecules was therefore of further interest. For this purpose, Eosin Y and Sudan IV were chosen as they exhibited the most prominent affinity for the $\mathrm{MeOH}-$ rich and 5CB-rich phases, respectively. The phase transfer experiments were carried out as described above, but the cooling temperature was systematically varied from $-5{ }^{\circ} \mathrm{C}$ to $23{ }^{\circ} \mathrm{C}$. The results presented in Fig. 5 show that as the temperature of the phase transfer was decreased, the selectivity for each phase increased. As an example, the $\mathrm{MeOH}$-rich phase contained $34 \mathrm{vol} \% 5 \mathrm{CB}$ at $20{ }^{\circ} \mathrm{C}$ and only $12 \mathrm{vol} \%$ at $0{ }^{\circ} \mathrm{C}$. For temperature-induced phase extraction from monophasic isotropic to biphasic isotropic, the partitioning of both compounds followed the trend in composition as determined by the phase diagram. Interestingly a further increase in partitioning was observed when cooling into the biphasic isotropic-nematic region, in particular for Sudan IV which is predominantly soluble in the 5CB-rich phase. For comparison, the temperaturedependent extraction of Eosin Y and Sudan IV was also carried out for a binary mixture of $\mathrm{MeOH}$ with $\mathrm{CB} 15$, the nonnematogenic analogue to 5CB with a branched alkyl chain (Fig. S11, ESI $\dagger$ ). The temperature-dependent tunability of the partitioning was also observed in this case, yet over a more limited temperature range due to the low UCST $\left(3^{\circ} \mathrm{C}\right)$ and without the possibility to extract into the nematic phase.

We want to emphasise that our findings on selective and tunable extraction do not predominantly rely on the nematic phase of $5 \mathrm{CB}$. The binary mixture of nematogenic and non-nematogenic

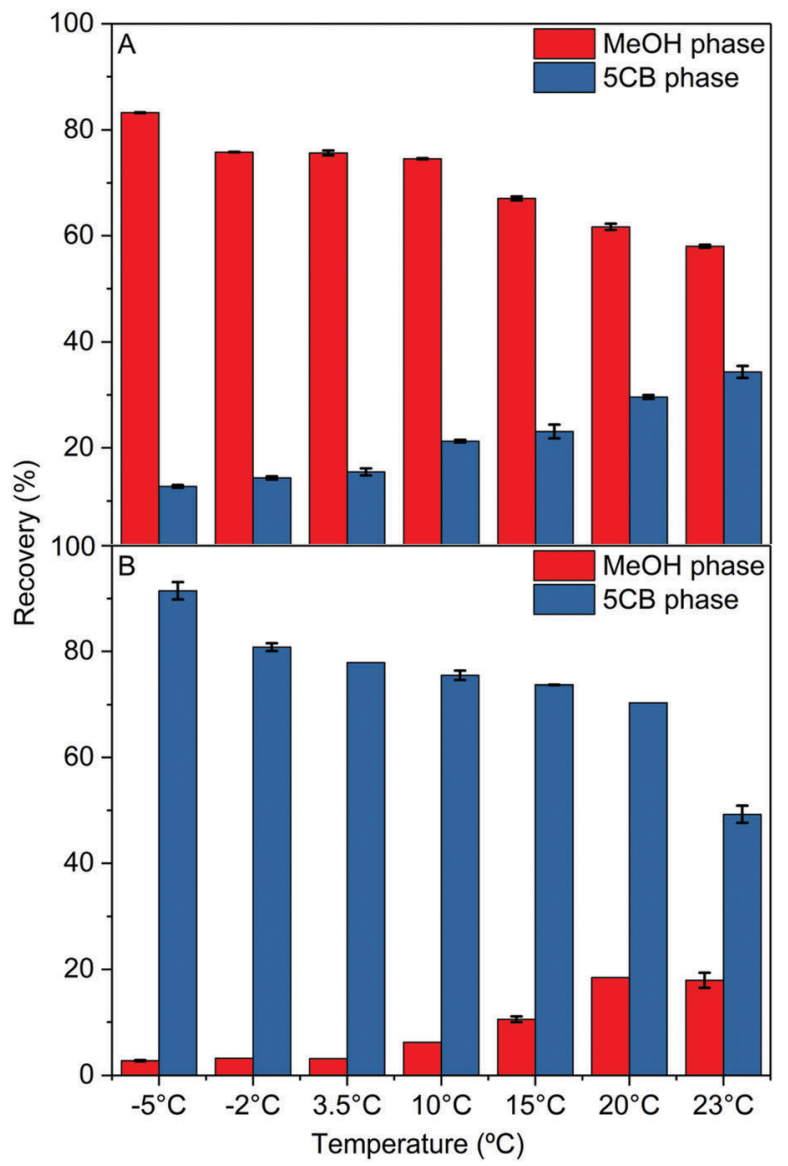

Fig. 5 Temperature dependence of molecular partitioning. Recovery into $\mathrm{MeOH}$-rich (red) and 5CB-rich (blue) phases at different temperatures for Eosin $Y(A)$ and Sudan IV (B).

compounds provides a complex solvent mixture that allows us to tune the phase content in the biphasic isotropic region over a vast compositional range and offers selective solvation. Beyond compositional tuning, further discrimination is accessible by extraction in the biphasic isotropic-nematic configuration at low temperatures.

While our findings suggest that the extraction is most efficient at the highest contrast in composition, we want to point out that the herein reported temperature-induced control over phase composition represents a useful tool in a practical context. For phase transfer-based sensing, the availability of the results at various temperatures increases experimental redundancy and thus may allow to reliably determine weakly selective sensing events with greater accuracy. ${ }^{44}$ One may also envision a situation where the multi-stage extraction of a number of compounds at different temperatures may allow greater versatility.

\section{Conclusions}

In conclusion, we report on the phase behaviour and possible applications of a binary liquid mixture of $\mathrm{MeOH}$ and the thermotropic LC 5CB. Four different phases were observed as a function of temperature and composition, namely monophasic 
isotropic, monophasic nematic, biphasic isotropic-isotropic and biphasic isotropic-nematic. At a $\mathrm{MeOH}$ volume content below $13 \%$, the non-nematogenic dilution allowed to finely tune the isotropic-to-nematic transition temperature from $35{ }^{\circ} \mathrm{C}$ to $1{ }^{\circ} \mathrm{C}$. Above 13 vol\% $\mathrm{MeOH}$ content, a binary mixture with an upper critical solution temperature of $24.4 \pm 0.5{ }^{\circ} \mathrm{C}$ was obtained, where the cooling temperature dictated the composition of both phases. The combination of selective solvation properties and temperature-induced compositional tuning represents a versatile platform for the extraction of target molecules and provides novel routes for sensing applications.

\section{Conflicts of interest}

There are no conflicts to declare.

\section{Acknowledgements}

This project received funding from the European Union's Horizon 2020 research and innovation programme under Grant Agreement No. 633635 (DIACHEMO). YY acknowledges University College London for an Overseas Research Scholarship. SGu is grateful for support by a start-up fund from the Department of Chemical Engineering at University College London. SGa acknowledges funding by the EPSRC (EP/K039229/1).

\section{References}

1 J.-L. Barrat and J.-P. Hansen, Basic Concepts for Simple and Complex Liquids, Cambridge University Press, 2003.

2 D. Balasubramanian and P. Mitra, J. Phys. Chem., 1979, 83, 2724-2727.

3 A. W. Francis, Critical Solution Temperatures, Adv. Chem. Series, American Chemical Society, 1961.

4 I. T. Horváth and J. Rábai, Science, 1994, 266, 72-75.

5 E. K. Paleologos, D. L. Giokas and M. I. Karayannis, Trends Anal. Chem., 2005, 24, 426-436.

6 J. G. Kralj, H. R. Sahoo and K. F. Jensen, Lab Chip, 2007, 7, 256-263.

7 S. B. Hawthorne, Yu. Yang and D. J. Miller, Anal. Chem., 1994, 66, 2912-2920.

8 P.-A. Albertsson, Separation of Cell Particles and Molecules, Wiley, 1990.

9 Y. Liu, R. Lipowsky and R. Dimova, Langmuir, 2012, 28, 3831-3839.

10 X. Han and D. W. Armstrong, Acc. Chem. Res., 2007, 40, 1079-1086.

11 M. G. Freire, A. F. M. Cláudio, J. M. M. Araújo, J. A. P. Coutinho, I. M. Marrucho, J. N. C. Lopes and L. P. N. Rebelo, Chem. Soc. Rev., 2012, 41, 4966-4995.

12 I. Buttinoni, G. Volpe, F. Kümmel, G. Volpe and C. Bechinger, J. Phys.: Condens. Matter, 2012, 24, 284129.

13 A. M. Menzel, Phys. Rep., 2015, 554, 1-45.

14 J. Beeckman, K. Neyts and P. J. M. Vanbrabant, Opt. Eng., 2011, 50, 81202.
15 Q. Li, Liquid Crystals Beyond Displays, John Wiley \& Sons, Inc., Hoboken, NJ, USA, 2012, pp. 569-573.

16 H. K. Bisoyi, S. Kumar, M. J. Green, A. L. Higginbotham, A. Sinitskii, D. V. Kosynkin, D. Tsentalovich, A. Nicholas, G. Parra-Vasquez, J. Schmidt, E. Kesselmann, Y. Cohen, Y. Talmon, J. M. Tour and M. Pasquali, Chem. Soc. Rev., 2011, 40, 306-319.

17 S. J. Woltman, G. D. Jay and G. P. Crawford, Nat. Mater., 2007, 6, 929-938.

18 P. G. de Gennes and J. Prost, The physics of liquid crystals, Clarendon Press, 1993.

19 G. W. Gray., K. J. Harrison and J. A. Nash, Electron. Lett., 1973, 9, 130-131.

20 R. L. Humphries and G. R. Luckhurst, Proc. R. Soc. London, Ser. A, 1976, 352, 41-56.

21 P. J. Flory, Adv. Polym. Sci., 1984, 59, 1-36.

22 M. Mucha, Prog. Polym. Sci., 2003, 28, 837-873.

23 E. R. Soule and A. D. Rey, Mol. Simul., 2012, 38, 735-750.

24 M. V. Gorkunov, G. A. Shandryuk, A. M. Shatalova, I. Yu Kutergina, A. S. Merekalov, Y. V. Kudryavtsev, R. V. Talroze and M. A. Osipov, Soft Matter, 2013, 9, 3578-3588.

25 J. Milette, V. Toader, E. R. Soulé, R. B. Lennox, A. D. Rey and L. Reven, Langmuir, 2013, 29, 1258-1263.

26 N. Kasch and I. Dierking, J. Chem. Phys., 2015, 143, 64907. 27 J. S. Dave and M. J. S. Dewar, J. Chem. Soc., 1954, 0, 4616.

28 W. Maier and A. Saupe, Z. Naturforsch. A, 1959, 14, 882-889. 29 W. Maier and A. Saupe, Z. Naturforsch. A, 1960, 15, 287-292.

30 F. Brochard, J. Jouffroy and P. Levinson, J. Phys., 1984, 45, 1125-1136.

31 A. Matsuyama and T. Kato, J. Chem. Phys., 1996, 105, 1654-1660.

32 C. C. Riccardi, J. Borrajo and R. J. J. Williams, J. Chem. Phys., 1998, 108, 2571-2575.

33 T. Araki and H. Tanaka, Phys. Rev. Lett., 2004, 93, 015701.

34 I. Teraoka, Polymer solutions: an introduction to physical properties, Jong Wiley \& Sons, Wiley, 2002.

35 M. V. Gorkunov and M. A. Osipov, Soft Matter, 2011, 7, 4348-4356.

36 J. W. Park and M. M. Labes, Mol. Cryst. Liq. Cryst., 1976, 34, 147-152.

37 S. DasGupta, P. Chattopadhyay and S. K. Roy, Phys. Rev. E, 2001, 63, 041703.

38 K. Denolf, G. Cordoyiannis, C. Glorieux and J. Thoen, Phys. Rev. $E, 2007$, 76, 051702.

39 T. M. Krygowski and M. K. Cyrański, Chem. Rev., 2001, 101, 1385-1420.

40 E. Huckel, Z. Phys., 1931, 70, 204-286.

41 S. Chicu, M. Munteanu, I. Cîtu, C. Şoica, C. Dehelean, C. Trandafirescu, S. Funar-Timofei, D. Ionescu and G. Simu, Molecules, 2014, 19, 9798-9817.

42 H. Hachisako, T. Yamazaki, H. Ihara, C. Hirayamab and K. Yamadaa, J. Chem. Soc., Perkin Trans. 1, 1994, 1681-1690.

43 W. O. Foye, T. L. Lemke and D. A. Williams, Foye's principles of medicinal chemistry, Wolters Kluwer Health/Lippincott Williams \& Wilkins, 2013.

44 L. D. Bonifacio, D. P. Puzzo, S. Breslav, B. M. Willey, A. McGeer and G. A. Ozin, Adv. Mater., 2010, 22, 1351-1354. 